\title{
Article
}

\section{Forecasting Solar Energetic Particle Events and Associated False Alarms}

Swalwell, Bill, Dalla, Silvia and Walsh, Robert William

Available at http://clok.uclan.ac.uk/28147/

Swalwell, Bill ORCID: 0000-0002-8411-8000, Dalla, Silvia ORCID: 0000-00027837-5780 and Walsh, Robert William ORCID: 0000-0002-1025-9863 (2018) Forecasting Solar Energetic Particle Events and Associated False Alarms. Proceedings of the International Astronomical Union, 13 (S335). pp. 324-327. ISSN 1743-9213

It is advisable to refer to the publisher's version if you intend to cite from the work. http://dx.doi.org/10.1017/S1743921317011036

For more information about UCLan's research in this area go to http://www.uclan.ac.uk/researchgroups/ and search for <name of research Group>.

For information about Research generally at UCLan please go to http://www.uclan.ac.uk/research/

All outputs in CLoK are protected by Intellectual Property Rights law, including Copyright law. Copyright, IPR and Moral Rights for the works on this site are retained by the individual authors and/or other copyright owners. Terms and conditions for use of this material are defined in the policies page.

\section{CLoK}

Central Lancashire online Knowledge www.clok.uclan.ac.uk

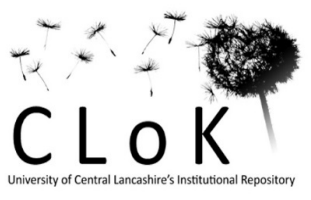




\title{
Forecasting Solar Energetic Particle Events and Associated False Alarms
}

\author{
Bill Swalwell ${ }^{1}$, Silvia Dalla ${ }^{1}$, and Robert Walsh ${ }^{1}$ \\ ${ }^{1}$ Jeremiah Horrocks Institute, University of Central Lancashire, \\ Preston, Lancashire, PR1 2HE, United Kingdom \\ email: bswalwell@uclan.ac.uk
}

\begin{abstract}
Because of the significant dangers they pose, accurate forecasting of Solar Energetic Particle (SEP) events is vital. Whilst it has long been known that SEP-production is associated with high-energy solar events, forecasting algorithms based upon the observation of these types of solar event suffer from high false alarm rates. Here we analyse the parameters of 4 very high energy solar events which were false alarms with a view to reaching an understanding as to why SEPs were not detected at Earth. We find that in each case there were present at least two factors which have been shown to be detrimental to SEP production.
\end{abstract}

Keywords. Sun: flares, coronal mass ejections, particle emission

\section{Introduction}

Solar Energetic Particles (SEPs) are a significant component of space weather. They may damage satellites, pose a radiation hazard to astronauts and humans in high-flying aircraft (particularly at high latitudes), and interfere with high-frequency communications' systems. Accurately forecasting their arrival at Earth has become vital.

It has long been known that the detection of SEPs at Earth is associated with solar flares which exhibit high emission in soft X-rays (SXR) and fast Coronal Mass Ejections (CMEs) (e.g. Dierckxsens et al. (2015)). The difficulty for SEP forecasting algorithms is, however, that SEPs are not detected at Earth following all such large solar events. For example, Klein et al. (2011) investigated all X class flares between longitudes W0 and $\mathrm{W} 90^{\circ}$ in the period 1996 to 2006, and they found that 30\% did not produce an enhancement of $>10 \mathrm{MeV}$ protons above the background level.

Figure 1 shows two plots of proton intensity as measured by the $\sim 40-80 \mathrm{MeV}$ energy channel of the Geostationary Orbital Environmental Satellites' (GOES) Energetic Particle Sensor (EPS) instruments. In Figure 1(a), a steep rise is seen following a magnetically well-connected large solar event which occurred on 17 May 2012 as may be expected; by contrast Figure 1(b) shows that a similarly large event which occurred on 18 March 2003 produced no rise at all. Such an event, which might reasonably have been expected to produce SEPs at Earth, may be termed a "false alarm". Here we examine 4 sample false alarm events with a view to determining why they failed to produce SEPs at Earth.

\section{False alarms for simple forecasting algorithms}

In Swalwell et al. (2017) we defined two simple SEP forecasting algorithms: the first, A.1, is based upon the observation of magnetically well-connected CMEs with a speed greater than 1,500 km/s ("fast CMEs"); the second, A.2, is based upon the observation of well-connected flares of class X. We compared the forecasts of each with historical data sets between January 1996 and March 2013. 


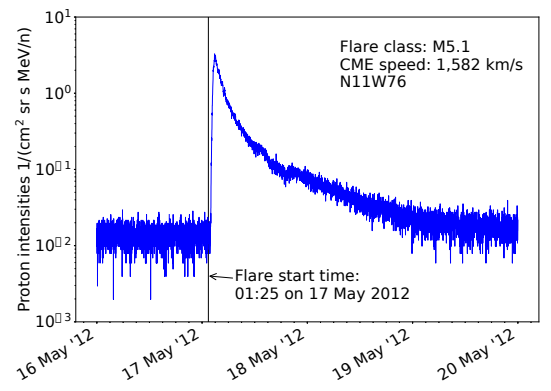

(a) A steep rise in energetic proton intensity is seen following a magnetically wellconnected M5.1 large solar event as may be expected.

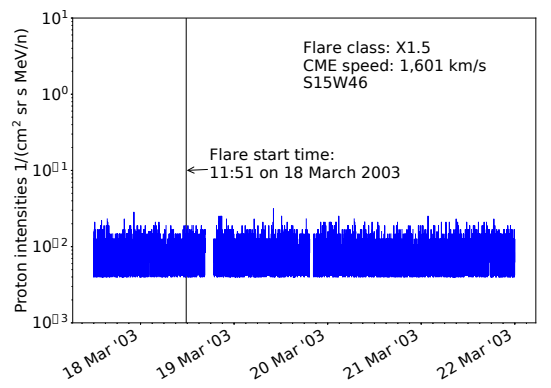

(b) A similarly large solar event produced no rise in energetic proton intensity at all. This event may be termed a "false alarm".

Figure 1. A comparison of $40-80 \mathrm{MeV}$ proton intensity following two different magnetically-well-connected large solar events.

Algorithm A.1 had a lower false alarm rate (28.8\%) than A.2 (50.6\%) but both missed a significant number of SEP events $(53.2 \%$ and $50.6 \%$ respectively). We determined that an algorithm which was based upon the parameters of both CMEs and flares produced better results than one based upon the observation of only one type of solar event.

We found a number of factors which are important to SEP production. Fast CMEs were less likely to produce SEPs if they were associated with a flare of class $<\mathrm{M} 3$, if their associated flare was of relatively short duration, and if they were not reported to be a halo. X class flares were less likely to produce SEPs if either they were not associated with a CME or were associated with a CME slower than $500 \mathrm{~km} / \mathrm{s}$, and if they were of relatively short duration (Swalwell et al. (2017)).

\section{Examples of false alarm events}

Table 1 gives four examples of high-energy solar events which it might have been thought would produce SEPs at Earth, but which failed to do so. Examination of some of their parameters sheds some light on why they were false alarms.

\subsection{Event 1: 1,813 km/s CME from N24W35 on 6 Jan 2000}

This was a very well magnetically-connected, very fast, CME. However, it was associated with a flare which was both short ( 21 minutes) and of relatively low class (C5.8). Furthermore, the CME itself was reported to have a width of just $67^{\circ}$.

\subsection{Event 2: X6.2 flare at N16E09 on 13 Dec 2001}

Towards the edge of the best magnetically-connected region, nevertheless this was such a large flare that it might have been expected to produce at least some enhancement of energetic protons. The fact that it did not may be connected to two parameters: (a) it was associated with a CME of relatively low speed $(864 \mathrm{~km} / \mathrm{s})$, and (b) it was of very short duration ( $\sim 15$ minutes).

\subsection{Event 3: X1.5 flare at S20W51 on 3 Jul 2002}

This very well magnetically-connected flare was associated with a very slow $(265 \mathrm{~km} / \mathrm{s})$ non-halo (width $261^{\circ}$ ) $\mathrm{CME}$, and was of very short duration ( $\sim 8$ minutes). 
Table 1. Example false alarms.

\begin{tabular}{cccc}
\hline Event no & Date & Event & Coordinates \\
\hline 1 & 6 Jan 2000 & CME speed 1,813 km/s & N24W35 \\
2 & 13 Dec 2001 & X6.2 flare & N16E09 \\
3 & 3 Jul 2002 & X1.5 flare & S20W51 \\
4 & 18 Mar 2003 & X1.5 flare associated with & S15W46 \\
\hline
\end{tabular}

3.4. Event 4: X1.5 flare with 1,601 km/s CME from $S 15 W 46$ on 18 Mar 2003

This is the event for which the energetic proton intensity is shown in Figure 1(b). It was extremely well magnetically-connected to Earth, and in this instance both the flare class and CME speed were high. Flare duration, however, was relatively short (at $\sim 29$ minutes) and the CME was reported to have a width of $263^{\circ}$.

\section{Conclusions}

Some very high-energy solar events may fail to produce SEPs at Earth even if they are very well magnetically-connected. Understanding why such events are false alarms may provide an insight as to which of their parameters are important to SEP production.

Swalwell et al. (2017) reported that fast CMEs associated with flares of class $<\mathrm{M} 3$ or of relatively short duration, and fast CMEs which were not reported to be a halo were more likely to be false alarms. X class flares not associated with a CME, or associated with a CME slower than $500 \mathrm{~km} / \mathrm{s}$ were more likely to be false alarms, as were those of relatively short duration.

Here we considered 4 sample false alarms. In each case 2 or more of the factors which were found to be detrimental to SEP production by Swalwell et al. (2017) were found to exist. In event number 1 there were 3 : the associated flare was relatively short $(\sim 21$ minutes); it was of class <M3 (C5.8); and the CME was not a halo (the width was $67^{\circ}$ ).

Event number 2 was a less well magnetically-connected flare (from E09) but at X6.2 it was very intense; event 3 was a lower class flare (albeit still large at X1.5) but very well connected. Neither produced SEPs but both had factors likely to result in a false alarm: each flare was short ( $\sim 15$ minutes and $\sim 8$ minutes respectively), and each was associated with a CME of relatively low speed $(864 \mathrm{~km} / \mathrm{s}$ and $265 \mathrm{~km} / \mathrm{s}$ respectively); and in the case of event 3 , the associated CME was not a halo.

Event number 4 illustrates that even a fast CME associated with a high intensity flare may sometimes be a false alarm. In this case, too, however the fact that the CME was not a halo and the short duration of the flare may explain why SEPs were not detected.

The full results and a more detailed analysis are presented in Swalwell et al. (2017).

\section{References}

Bentley, R. D., Csillaghy, A., Aboudarham, J., Jacquey, C., Hapgood, M. A., Bocchialini, K., Messerotti, M., Brooke, J., Gallagher, P., Fox, P., Hurlburt, N., Roberts, D. A., \& Duarte, L. S. 2011, Advances in Space Research, 47, 2235-2239

Dierckxsens, M., Tziotziou, K., and Dalla, S., Patsou, I., Marsh, M., Crosby, N., Malandraki, O., \& Tsiropoula, G. 2015, Solar Physics, 290, 841-874

Gopalswamy, N., Yashiro, S., Michalek, G., Stenborg, G., Vourlidas, A., Freeland, S., \& Howard, R. 2009, Earth Moon and Planets, 104, 295-313

Klein, K.-L., Trottet, G., Samwel, S., \& Malandraki, O. 2011, Solar Physics, 269, 309-333

Swalwell, B., Dalla, S., \& Walsh, R. 2017, Solar Physics, submitted 